\title{
The ethics of regenerative medicine
}

\author{
Göran Hermerén ${ }^{1}[0$
}

Received: 2 March 2020 / Accepted: 26 January 2021 / Published online: 20 February 2021

(c) The Author(s) 2021

\begin{abstract}
Most developments in regenerative medicine have in common that there are many uncertainties and knowledge gaps. These features make the evaluation of long-term consequences of the available options difficult and have consequences for the ethical issues raised. This paper presents an overview of ethical issues raised in regenerative medicine, using as a starting point a list of stakeholders and their interests. Ethical issues are introduced via a simplified account of a project that focuses on several difficult problems, as well as a conceptual framework consisting of the following key concepts: present situation, goals, difficulties on the road toward the goals, and strategies for dealing with the difficulties. The list of ethical issues discussed includes safety and efficacy, patient consent, information, professional responsibilities, as well as equity and fairness. The issues and the underlying values need to be clarified, specified, debated, and ranked in order of importance. A particular problem is that values sometimes clash: Certain values can be achieved only at the expense of others. If and when values clash, principles are available that can guide the decision making. The paper comments on two such principles with implications for the particular issue of patient access to experimental treatments: the precautionary principle and the principle of proportionality. The paper ends with some conclusions for the future.
\end{abstract}

Keywords Ethics $\cdot$ Regenerative medicine $\cdot$ Value conflicts $\cdot$ Precautionary principle $\cdot$ Principle of proportionality

\section{Introduction}

Regenerative medicine has been defined (Cossu et al. 2018) as an emerging medical endeavor aimed at tissue regenerations via small molecule drugs, biological therapies, medical or tissue-engineered devices, or cells and genes. The objective is to repair human tissue and organs.

Partly different ethical problems are raised in different areas of regenerative medicine (Hyun 2013). Moreover, different ethical challenges are raised as the scientific landscape changes; thus, a historical dimension is relevant. The moral status of the embryo was once the central issue in the discussion of human embryonic stem cell research. It has now been replaced by a number of other more specific problems, though advances in stem cell research do not obviate the need for human embryonic stem cells (Hyun et al. 2007).

More specific issues include those raised by the long-term consequences of gene editing, research and development of

Göran Hermerén

goran.hermeren@med.lu.se

1 Department of Medical Ethics, Biomedical Centre, Lund University, Lund, Sweden chimaeras, conditional approval for marketing license or discussion of the 14-day limit for research on human embryos, as well as early patient access to experimental treatment; on some of these issues, see, for instance, (Sipp 2015; Hyun et al. 2016; Daley, et al. 2019; Hermerén 2015; Koplin and Wilkinson 2019; Porsdam Mann et al. 2019).

Most developments in regenerative medicine have in common that there are many uncertainties and knowledge gaps. These features make the evaluation of long-term consequences of the available options difficult and have consequences for the ethical issues raised.

\section{Stakeholders and their interests}

An overview of the stakeholders and their interests will provide a basis for any normative discussion how their interests should be valued, and about what should be permitted, praised, or prohibited.

The list of stakeholders is long and includes present and future patients, their relatives and families, physicians, clinics, healthcare services, medical journals, those in the product supply chain, researchers, funding organizations, 
professional organizations, regulators, policy makers, and taxpayers.

To begin with, what do the stakeholders want to achieve? We cannot assume that they all want the same. What do they want to avoid? In the long and short run? To answer such questions, the stakeholders must be identified and their interests indicated and studied. This could be clarified by empirical research, though such research will not settle any normative or ethical issues.

There is a variety of values underpinning the interests of the stakeholders. We may want to-as in the general framework suggested by the US National Academies, Engineering and Medicine (2019), with my comments in italics:

Promote societal value, but then these values need to be specified.

Minimize negative societal impact (which also needs to be specified).

Protect the interests of research participants (if they cannot do so themselves).

Advance the interests of patients, though these may differ.

Maximize scientific rigor and data quality.

Engage relevant communities-but which ones are relevant?

Ensure oversight and accountability—but how?

Recognize appropriate governmental and policy role, but views may differ on what is appropriate in this context.

This overview can be used as a starting point to identify the key ethical issues raised. There is a need to be specific also about values and goals-if we want to avoid talking at cross purposes.

\section{The landscape}

Let me begin the overview of a landscape by giving a simplified account of a project that focuses on several difficult problems, among others the following ones:

When is it justified to withhold a potentially successful treatment in randomized placebo controlled trials, particularly in phase III, to study the safety, efficacy, and repeatability of the use of MSCs (mesenchymal stromal cells) to repair damaged organs in patients suffering from graft-versus-host disease? When is it justified to involve children in such trials, if the benefit of a potentially experimental treatment amounts to potentially life-saving therapy and the efficacy of the "best proven" therapy is poor?

The main ethical issues here include:

(1) Risk-benefit analysis and management; who decides when the risk-given the chance to benefit-is worth taking? What about criteria for selection of patients and how to deal with desperate patients? How to avoid hype and provide justified hope?
(2) Information to and consent from whom? Conditions for carrying out RCTs with severely ill patients, conditions for de-blinding, for involving vulnerable groups such as children in randomized placebo controlled trials; gray areas in inclusion and exclusion criteria; use of placebo; clarification of "best proven therapy."

It is useful in the ethical analysis to describe the landscape using four concepts: the current situation (where are we?), the goals (where do we want to be?), the obstacles on the road toward the goals, and strategies for dealing with these obstacles.

The current situation is the obvious starting point for any discussion of desirable changes. This includes the clinical and regulatory context as well as evolving business models-where patients sometimes have to pay to be enrolled in clinical trials. An important concern expressed in the coming joint EASAC (European Academies Science Advisory Council) and FEAM (Federation of European Academies of Medicine) report (EASAC 2020) is that some companies are advancing therapies with poor efficacy—and perhaps inducing patient organizations to lobby for marketing authorization-that result in high cost for health care systems.

Let me continue with some basic points about ethics. First, well-founded decisions in this area are based on evidence of two kinds: values and knowledge. The latter I will provisionally define as justified beliefs and refer to in what follows as (scientific) evidence.

The ethical problem at hand-typically a clash of values-will indicate what evidence is relevant. It may include the health condition of patients, their diagnosis, possible therapeutic options, costs, along with knowledge about regulations, patient attitudes, as well as specific information about, for instance, the origin of the cells used, the characteristics of the cells, effects of previous trials, documentation of clinical evidence on efficacy and side effects, and the number of patients treated previously with the same procedure.

In order to arrive at ethically acceptable conclusions in the long-range interest of all stakeholders involved, a robust scientific basis is needed along with values that are debated, clarified, specified, and ranked in importance.

\section{Knowledge-related goals}

The general goal is to improve the quality of the evidence base. To achieve this, more rigorous research is needed, including carefully designed and conducted clinical trials. Clinical research is expensive and requires thorough ethical review as well as commitment of research sponsors.

Better knowledge base might also be achieved by improving publication practices, by promoting research integrity, by publishing also outcomes of failed trials, by avoiding 
publication bias and selective reporting of the outcomes of regenerative medicine trials-but then we are already in the domain of research ethics and research integrity.

Patients, clinicians, and regulators need access to more and better scientific evidence. It is both a scientific and ethical obligation to reduce the uncertainties and, if possible, fill the knowledge gaps. This is essential to be able to provide a better risk-benefit analysis and more accurate information to patients and research subjects.

"Selective" reporting or "overselling" the results on efficacy is often dictated by the need of a positive result to raise further funds. This is sometimes said to be justified by the future development, only possible with these funds, of a more efficacious and safer medicinal product. Recent history, however, shows that an early and not fully justified marketing authorization rarely leads to the development of such a product.

The approach, to justify hype and overselling of results on efficacy, is in my view not ethically acceptable. I would rather be inclined to argue for changes in the funding policy, against too much focus on the practical outcomes of the research, and for better understanding of the need for more basic research in order to arrive at efficacious and safe medical products.

\section{Value-related goals}

Let me begin with a simplified research example to make the discussion a bit more concrete.

The project is investigating a novel SC therapy for severe osteogenesis imperfecta, OI, a currently untreatable and severe genetic condition whereby affected fetuses and children develop life-threatening skeletal pathology. A potential therapeutic intervention will be given to an individual affected by severe OI, either to fetuses or to infants. The study also involves pregnant women who agree to donate fetal tissue for isolation of fetal liver-derived mesenchymal stem cells, MSCs, used for the intervention. Only fetuses or infants most severely affected by OI will be eligible to take part in the study.

The issues raised by the project include: What are the risks of miscarriage or harm to the fetus? Which are the therapeutic benefits? How certain are they? Who should weigh risks against benefits? Who decides, who may veto, in view of many uncertainties? What should be the role of the various stakeholders involved? What efforts should be made to ensure a free and informed consent? By whom from whom?

Against the background of such an example, the list of ethical issues would include.

Safety and efficacy.

Patient consent.

Information.
Professional responsibilities.

Equity and fairness.

The keywords above refer to value-related goals, or goals which are underpinned by values. Safety and efficacy are good things, to be pursued, like equity and fairness, so is patient consent which presupposes relevant and understandable information. Behind and motivating a list of personal responsibilities, there are values; those who neglect their responsibilities can cause harm to patients as well as to serious research.

But these values deserve some comments. They need to be clarified, specified, debated, and ranked in order of importance. First some general comments, then I will mention a few difficulties and obstacles on the road.

Safety and efficacy Assessing the efficacy and safety of a treatment is a challenge when there is limited research evidence and little or no clinical experience of use, creating high levels of uncertainty. Factors that might be undetermined include the appropriate dosage and other interventions required to make a drug safe and effective, particularly concerning long-term effects of the use of novel technologies that intervene in the brain.

In a Bioethics Briefing, the Nuffield Council (2018) wrote: "For patients who have limited options, uncertainty about safety and efficacy may be outweighed by the possibility, even if very slight, that the treatment could be effective for them" (page 4). What is a rational decision in such a situation, and who is to decide about it? Obviously, healthcare professionals have an important responsibility to help patients to a decision that is as informed as possible, and a decision they can live with.

Patient consent The rapid development of research, the uncertainties and the knowledge gaps, the difficulty to foresee future uses of obtained data, donated cells and tissues have also started a discussion of different forms of consent, where one distinction is between opt in and opt out. According to the first, opt in: If you have not said yes to the request, you have said no. According to the second, opt out: If you have not said no, you have said yes.

The discussion has continued with other forms of consent, including broad consent: a consent model that allows for current and future access and use of samples or data for research without necessarily specifying what the focus of such studies might be. This is different from tiered consent: a consent model in which participants are given a set of options allowing them to select how they want to participate in the research. It is also different from dynamic consent, a consent model in which participants are able to alter their preferences over time. The extent to which regulatory and oversight committees would accept these new consent models is still not quite clear. GDPR, the General Data Protection Regulation, may be interpreted as opening up for a somewhat broader consent 
than was generally accepted by many ethics committees in the past.

Information Information sources need to be critically assessed-but also, and in particular-the contents. It is essential that the information provided is relevant, correct, understandable, and not misleading. The challenge to achieve this is often underestimated. Many difficulties are well known from research in the social and behavioral sciences (Kahneman 2012). Special problems arise when the patient is a minor or a mentally incapacitated adult not able to give consent.

The many uncertainties and knowledge gaps complicate the decision making and the information procedure because the risks (and benefits) may be partly unknown. But this also underlines the previous conclusion: There is an ethical as well as a scientific duty to improve the evidence.

Equity and fairness Patient access to experimental treatment is unequal and limited by considerable costs. This is a problem for patients, their families, and health services (EASAC 2020). Moreover, since resources for medical research and health care are limited, it means that using the resources for one particular purpose means that they cannot be used for some other purpose. Well-founded decisions here require information about long-term consequences, particularly concerning safety, efficacy, and costs of the various alternative options. Many gaps and uncertainties are a challenge for the decision maker.

Order between issues There is a natural order between these issues. In my view, risk-benefit analysis is basic, for without an appropriate risk-benefit analysis it is not possible to inform regulators, patients or research subjects in an adequate way. Without adequate information, it is not possible to obtain a consent worth much. But already here there is an intriguing interplay between science and values. When a decision is taken that the risk-benefit ratio is favorable, that the risk is worth taking, this decision is also based on values.

The same holds for decisions about safety levels. This suggests the following conclusion: Decisions about safety level and early access to experimental treatments are not value neutral or ethically neutral. A high safety level favors some at the expense of others; a low safety level is likely to exploit others (present patients and research subjects) and may benefit future patients.

For "first in human" trials, safety has to be established in phase I. Therefore, there is an unavoidable risk represented by adverse events that have not and could not be foreseen at the time of trial planning and may not be present or detectable in pre-clinical animal studies. Patients should also be informed of this uncertainty.

\section{Difficulties and strategies}

What are the particular obstacles on the road, and what strategies can be used to deal with them with some chance of success?

A general difficulty is that often we do not know enough about the various options, their alternatives, and consequences. Both alternatives and consequences need to be identified, specified, and valued. There are also uncertainties and knowledge gaps about the values of the stakeholders, about their short-term or long-term goals. Their values have not been enough clarified, specified, or ranked in importance.

A number of more specific obstacles or difficulties are discussed in the joint EASAC and FEAM report (EASAC 2020).

What strategies could be used to deal with them? A strategy for dealing with the uncertainties and knowledge gaps of the value landscape mentioned in EASAC (2020) is tackling gaps in training on ethical, legal, and societal issues in regenerative medicine, including how to involve other stakeholders, especially patients, in research design and review.

Some of these obstacles can thus be dealt with by engaging with the public and patients in order to counter misinformation; the work of EuroStemCell, the ISSCR Patient Handbook (2008), and the updated ISSCR Guidelines (2016) can be mentioned as good examples. Behavioral and social science research as well as ethical analysis of the various value premises in the debate can provide further food for thought.

Unfair and limited access to therapies and experiments treatments could be dealt with by ensuring that patients do not have to pay to be enrolled in clinical trials.

Any further particular problems?

A particular problem is that values sometimes clash: Certain values can be achieved only at the expense of others. In order to resolve such conflicts, research is needed so that the values and goals can be specified and debated. The reason is that many of the values-even when clarified and specified - are relevant and legitimate. The issue then concerns their relative importance.

Examples are not too difficult to find. Sometimes, new knowledge can be achieved only if certain risks regarding patient safety are taken. So what is more important: New knowledge or safety? Patients, particularly if they or their children are in a desperate situation, may consent to participating in high-risk trials or trials with unknown efficacy. So what is more important: Safety, efficacy or consent?

Early access to expensive treatments may require that resources are diverted from elsewhere in health services to novel treatments without good evidence of their benefit. 
So what is more important: Uncertain investment in a treatment of so far unmet medical needs that may be costeffective in the future or using the resources for treatment of less dramatic conditions where the benefits are known?

There is no simple answer, but the question requires analysis and debate-in light of two principles discussed in the next section.

\section{Two principles}

Ethical problems are raised by conflicting values. If and when values clash, principles are available that can guide the decision making. I will here call attention to two such principles with implications for the particular issue of patient access to experimental treatments: the precautionary principle and the principle of proportionality.

The precautionary principle, strictly interpreted, requires us to stop if there are uncertainties about the risks involved, and it places the burden of proof of safety on those who want to promote a change. But the principle of proportionality is more open, as it boils down to four conditions (Hermerén, 2012), which at all times can be discussed, assessed, argued for, and applied in light of the present evidence. Decisions can then be taken which are not carved in stone but can be changed as the evidence and value landscape changes.

This suggests an approach encouraging an ongoing overview of processes in light of changing evidence and values within restrictions imposed e $\mathrm{g}$ by respect for human rights and concern for animal welfare. Moreover, the principle of proportionality can be applied in very different contexts, and it is important to make explicit what is taken for granted in each context.

What can we learn from the history of medicine? We can learn that willingness to take some calculated risks has sometimes been necessary for the advancement of new knowledge and improved diagnostic and therapeutic methods. To insist on zero risk would halt progress-and limit possibilities to help patients in the future.

\section{Conclusions for the future}

There is more to be done to train researchers in regenerative medicine on the ethical, legal, and social issues (Illes et al. 2017). There is also a need to do more to understand and clarify the responsibilities of stakeholders such as the research funding organizations, physicians and health services, regulators, medical journals, those in the product supply chain, and patients and their families.

What remains to be further clarified? What actions need to be taken by whom and when? One such issue, mentioned toward the end of EASAC (2020), is this: Tissue and cell legislation is based on principles of altruistic donation, and the commercialization of the donated biological material, once it becomes classified as a medicinal product, needs to be addressed-with transparent information and rules. Who gets paid for what—under what conditions?

Funding Open access funding provided by Lund University.

\section{Compliance with ethical standards}

Conflict of interest The author declares that he has no conflict of interest.

Open Access This article is licensed under a Creative Commons Attribution 4.0 International License, which permits use, sharing, adaptation, distribution and reproduction in any medium or format, as long as you give appropriate credit to the original author(s) and the source, provide a link to the Creative Commons licence, and indicate if changes were made. The images or other third party material in this article are included in the article's Creative Commons licence, unless indicated otherwise in a credit line to the material. If material is not included in the article's Creative Commons licence and your intended use is not permitted by statutory regulation or exceeds the permitted use, you will need to obtain permission directly from the copyright holder. To view a copy of this licence, visit http://creativecommons.org/licenses/by/4.0/.

\section{References}

Cossu G, Birchall M, Brown T et al (2018) Commission: stem cells and regenerative medicine. Lancet 391:883-910

Daley GQ, Lovell-Badge R, Steffann J (2019) After the storm-a responsible path for genome editing. N Engl J Med 380:897-899

EASAC (2020). Challenges and potential in regenerative medicine. A joint report from EASAC, European Academies' Science Advisory Council, and FEAM, Federation of European Academies of Medicine. To be published in the spring of 2020.

Hermerén G (2012) The principle of proportionality revisited: interpretations and applications. Med Health Care Philos 15:373-382

Hermerén G (2015) Ethical considerations in chimera research. Development 142:3-5

Hyun I (2013) Bioethics and the future of stem cell research. Cambridge University Press, Cambridge

Hyun I, Hochedlinger K, Jaenisch R, Yamanaka S (2007) New advances in iPS cell research do not obviate the need for human embryonic stem cells. Cell Stem Cell 1:367-368

Hyun I, Wilkerson A, Johnston J (2016) Revisit the 14-day rule. Nature 533:169-171

Illes J, Sipp D, Kleiderman E (2017) A blueprint for the next generation of ELSI research, training and outreach in regenerative medicine. Regener Med 2:21

ISSCR (2008) Patient Handbook on Stem cell therapies. Available at www.isscr.org/docs/default-source/patient- handbook/isscrpatienthandbook.pdf

ISSCR (2016) Guidelines for Stem Cell research and clinical translation. Available at www.isscr.org/policy/guidelines-for-stem-cellresearch-and-clinical-translation

Kahneman D (2012) Thinking fast and slow. Penguin Books, London

Koplin J, Wilkinson D (2019) Moral uncertainty and the farming of human-pig chimeras. J Med Ethics 45:440-446

National Academies of Sciences, Engineering and Medicine (2019) Framework for addressing ethical dimensions of emerging and 
innovative biomedical technologies: a synthesis of relevant National Academies reports. The National Academies Press, Washington, DC. https://doi.org/10.17226/25491

Nuffield Council on Bioethics (2018) Patient access to experimental treatments. Bioethics briefing, published 20/11/2018. Nuffield Council on Bioethics, London.
Porsdam Mann S, Sun R, Hermerén G (2019) A framework for the ethical assessment of chimeric animal research involving human neural tissue. BMC Med Ethics 20:10. https://doi.org/10.1186/ s12910-019-0345-2

Sipp D (2015) Japan lowers the bar for regenerative medicine products. Cell Stem Cell 16:353-356 